\title{
Selective Ultrafast Probing of Transient Hot Chemisorbed and Precursor States of CO on Ru(0001)
}

M. Beye, ${ }^{1,2}$ T. Anniyev, ${ }^{1}$ R. Coffee, ${ }^{3}$ M. Dell'Angela, ${ }^{4}$ A. Föhlisch,${ }^{2,5}$ J. Gladh,${ }^{6}$ T. Katayama, ${ }^{1}$ S. Kaya, ${ }^{1}$ O. Krupin, ${ }^{3,7}$ A. Møgelhøj, ${ }^{8,9}$ A. Nilsson, ${ }^{1,6,8,10}$ D. Nordlund, ${ }^{10}$ J. K. Nørskov, ${ }^{8,11}$ H. Öberg, ${ }^{6}$ H. Ogasawara, ${ }^{10}$ L. G. M. Pettersson, ${ }^{6}$ W. F. Schlotter, ${ }^{3}$ J. A. Sellberg, ${ }^{1,6}$ F. Sorgenfrei, ${ }^{4}$ J. J. Turner, ${ }^{3}$ M. Wolf, ${ }^{12}$ W. Wurth, ${ }^{4}$ and H. Öström ${ }^{6, *}$

${ }^{1}$ SIMES, SLAC National Accelerator Laboratory, 2575 Sand Hill Road, Menlo Park, California 94025, USA

${ }^{2}$ Institute for Methods and Instrumentation in Synchrotron Radiation Research, Helmholtz-Zentrum Berlin für Materialien und Energie GmbH, Wilhelm-Conrad-Röntgen Campus, Albert-Einstein-Strasse 15, 12489 Berlin, Germany

${ }^{3}$ Linac Coherent Light Source, SLAC National Accelerator Laboratory, 2575 Sand Hill Road, Menlo Park, California 94025, USA

${ }^{4}$ University of Hamburg and Center for Free Electron Laser Science, Luruper Chausse 149, D-22761 Hamburg, Germany

${ }_{5}^{5}$ Institut für Physik und Astronomie, Universität Potsdam, Karl-Liebknecht-Straße 24-25, 14476 Potsdam, Germany

${ }^{6}$ Department of Physics, AlbaNova University Center, Stockholm University, SE-10691 Stockholm, Sweden

${ }^{7}$ European XFEL GmbH, Albert-Einstein-Ring 19, 22761 Hamburg, Germany

${ }^{8}$ SUNCAT Center for Interface Science and Catalysis, SLAC National Accelerator Laboratory, 2575 Sand Hill Road, Menlo Park, California 94025, USA

${ }^{9}$ Department of Physics, CAMD, Technical University of Denmark, DK 2800 Lyngby, Denmark

${ }^{10}$ Stanford Synchrotron Radiation Lightsource, SLAC National Accelerator Laboratory, 2575 Sand Hill Road, Menlo Park, California 94025, USA

${ }^{11}$ Department of Chemical Engineering, SUNCAT Center for Interface Science and Catalysis, Stanford University, Stanford, California 95305, USA

${ }^{12}$ Fritz-Haber Institute of the Max-Planck-Society, Faradayweg 4-6, D-14195 Berlin, Germany

(Received 1 February 2013; revised manuscript received 3 April 2013; published 1 May 2013)

\begin{abstract}
We have studied the femtosecond dynamics following optical laser excitation of $\mathrm{CO}$ adsorbed on a $\mathrm{Ru}$ surface by monitoring changes in the occupied and unoccupied electronic structure using ultrafast soft x-ray absorption and emission. We recently reported [M. Dell'Angela et al. Science 339, 1302 (2013)] a phonon-mediated transition into a weakly adsorbed precursor state occurring on a time scale of $>2 \mathrm{ps}$ prior to desorption. Here we focus on processes within the first picosecond after laser excitation and show that the metal-adsorbate coordination is initially increased due to hot-electron-driven vibrational excitations. This process is faster than, but occurs in parallel with, the transition into the precursor state. With resonant x-ray emission spectroscopy, we probe each of these states selectively and determine the respective transient populations depending on optical laser fluence. Ab initio molecular dynamics simulations of $\mathrm{CO}$ adsorbed on $\mathrm{Ru}(0001)$ were performed at 1500 and $3000 \mathrm{~K}$ providing insight into the desorption process.
\end{abstract}

DOI: 10.1103/PhysRevLett.110.186101

A microscopic understanding of surface reactions is a longstanding goal in heterogeneous catalysis, which eventually would allow tailoring catalysts for optimal control and efficient use of energy and precious resources. A key to achieving such a deeper understanding of surface reactions would be the selective real-time probing of the dynamic evolution of the adsorbate electronic structure of different transient species during chemical reactions. This is realized in the present study, where intermediates in different bonding configurations are selectively characterized and thus a reaction pathway is determined.

The prime technique for real-time probing of surface reactions has so far been time-resolved vibrational spectroscopy, such as surface sensitive nonlinear optical sumfrequency generation spectroscopy (SFG). This technique has been successfully utilized, e.g., to monitor transient excitation of external adsorbate vibrational modes that couple to diffusion [1] and to desorption reactions [2-4].
PACS numbers: 68.35.Ja, 78.47.J-, 78.70.En, 82.20.-w

In particular, excitation of frustrated rotations was observed during the fs-laser-induced desorption process of $\mathrm{CO}$ from $\mathrm{Ru}(0001)$ [2]. Here we build on those findings and directly study the transient evolution of the electronic structure.

The Linac Coherent Light Source (LCLS) x-ray freeelectron laser (FEL) provides intense femtosecond $\mathrm{x}$-ray pulses, which allow using the atom sensitivity of soft x-ray spectroscopies to probe dynamics induced by an optical laser. Thus we achieve both the chemical sensitivity and temporal resolution relevant for real-time probing of the electronic structure during molecular transformations. Recently it was shown how an optical laser pump combined with ultrafast $\mathrm{x}$-ray absorption (XAS) and emission (XES) spectroscopy as time-resolved probes using LCLS $\mathrm{x}$-ray pulses could detect a weakly adsorbed precursor state that appears transiently during $\mathrm{CO}$ desorption a few picoseconds after the optical laser has excited the sample [5]. Here we demonstrate essential adsorbate dynamics 
of a different nature on the much shorter subpicosecond time scale.

XAS and XES are well established powerful tools for studying surface chemical bonding under equilibrium conditions due to their element specificity that allows essentially background-free probing of the adsorbate valence electronic structure [6,7]. In XES, x-ray absorption resonances can be used to selectively enhance the signal for molecules in a particular chemical environment and thus to specifically probe the valence electronic structure of a particular species among those that coexist at the surface $[8,9]$.

In the present study we combine ultrafast XAS, that probes the dynamics of all adsorbed molecules, with ultrafast resonant XES that selectively probes different subsets of the molecules. We observe vibrationally excited chemisorbed molecules coexisting with precursor adsorbed $\mathrm{CO}$ and unify the picture of the desorption process from SFG with that of transient population of the precursor state from XAS and XES. In particular, we find that by changing the incoming photon energy for XES, we can selectively probe $\mathrm{CO}$ molecules in the precursor state and discriminate them from $\mathrm{CO}$ molecules with an increased coordination to more than one metal atom corresponding to $\mathrm{CO}$ in a vibrationally hot chemisorbed state with large translational amplitudes. Note that the $\mathrm{x}$-ray photon energy only affects which subensembles are probed, not the actual population of these. This higher coordination state occurs already at short time scales $(<1 \mathrm{ps})$ prior to the transient trapping in the precursor state and the two states coexist at longer time scales. The vibrationally hot chemisorbed state provides the initial (fast) pathway towards desorption as is further corroborated by $a b$ initio molecular dynamics (AIMD) simulations.

The experiments were performed at the soft x-ray (SXR) beam line at LCLS [10]. A CO adlayer on $\mathrm{Ru}(0001)$ was excited using $400 \mathrm{~nm}$ optical laser pulses with a duration of $50 \mathrm{fs}$ at an absorbed optical laser fluence of 100 or $140 \mathrm{~J} / \mathrm{m}^{2}$, which leads to roughly $10 \%$, respectively $30 \%$, desorption yield following a single laser shot [11]. The $\mathrm{Ru}(0001)$ crystal was cleaned by standard procedures [5] and kept at $300 \mathrm{~K}$. It was continuously scanned during the experiment and redosed via a background pressure of $\mathrm{CO}$ at a partial pressure of $1 \times 10^{-8}$ torr to ensure a fresh surface layer for each laser shot. The photon energy of the $100 \mathrm{fs}$ FEL pulses was scanned across the oxygen $1 \mathrm{~s} \rightarrow$ $2 \tilde{\pi}^{*}$ resonance in the range of $531-535 \mathrm{eV}$ for both XAS and resonant XES experiments; the tilde distinguishes molecular orbitals in the chemisorbed state from their gas phase equivalents and indicates that they are modified through orbital mixing to form the surface chemical bond.

The AIMD simulations were performed at 1500 and $3000 \mathrm{~K}$ on a model system with one or two CO molecules adsorbed on a $(2 \times 2)$ unit cell of a $\mathrm{Ru}(0001)$ slab consisting of three layers [12]. The forces were calculated using density functional theory (DFT) [13] and the revised
Perdew-Burke-Ernzerhof (RPBE) functional [14] that describes the chemisorbed state accurately but cannot describe a weakly bound precursor state that is obtained only through van der Waals interactions [15]. To prepare the initial condition the substrate was equilibrated at the target temperature, while the $\mathrm{CO}$ was kept at $300 \mathrm{~K}$ in the equilibrium on-top adsorption site. The system was then released and the evolution followed in time for up to $20 \mathrm{ps}$. We also performed two-temperature model calculations $[16,17]$, which do not include direct photoexcitations, but capture the essential substrate electron-phonon dynamics during thermal equilibration of the system.

The chemical bonding of $\mathrm{CO}$ chemisorbed to metal surfaces has previously been studied in detail using XES and DFT [6,18,19] and occurs mainly via interaction between the molecular $\pi$ system and the metal $d$ levels, which leads to a unique spectroscopic signature in the form of new electronic states, denoted $\tilde{d}_{\pi}$, in the spectral region between the $1 \tilde{\pi}$ and $2 \tilde{\pi}^{*}$ orbitals, among other changes. The top panel (a) in Fig. 1 shows the $\mathrm{O} K$-edge XE and XA spectra of $\mathrm{CO}$ chemisorbed on the $\mathrm{Ru}(0001)$ surface before arrival of the optical pump laser pulse and shows the typical features expected for adsorbed CO [20]. The XE

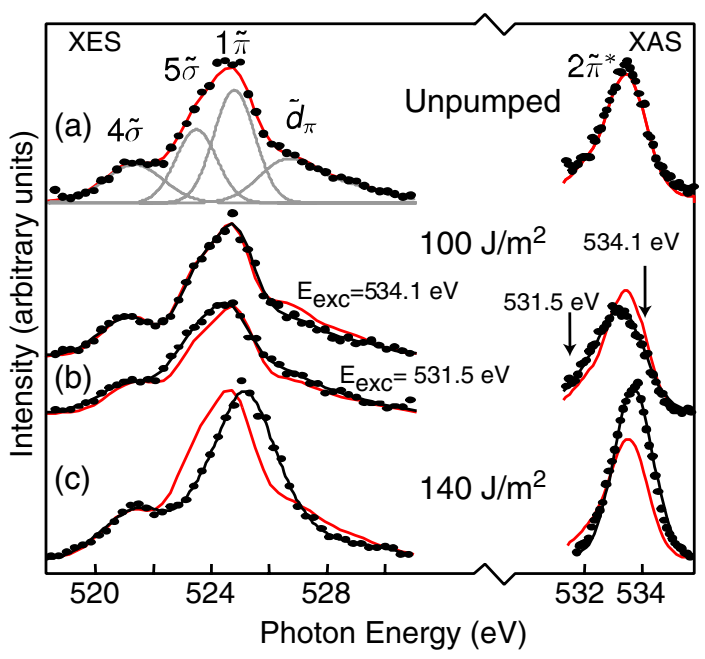

FIG. 1 (color online). Spectral distributions for characteristic states during $\mathrm{CO}$ desorption. On the left, selectively probed $\mathrm{O} 1 \mathrm{~s}$ $\mathrm{x}$-ray emission spectra are shown, whereas on the right the corresponding ensemble averaged $\mathrm{O} 1 s \mathrm{x}$-ray absorption spectra are displayed. (a) Unpumped spectra at negative pump-probe delay (i.e., before the optical laser pump pulse arrives) together with a spectral decomposition (grey) into the molecular orbitals. The spectra in (b) and (c) are averaged over the delay range between 2 and 15 ps (the appropriate spectra at negative delay are overlaid in red). (b) At lower optical laser fluence, we observe a shifted XAS resonance. Absorbing x-ray photons in the red or blue shoulder of the resonance as indicated on the right, we pick up different spectral signatures in XES stemming from different subsets of the molecules (left). (c) Corresponding spectra for high optical laser fluence. Note that the relative scaling of XES and XAS is arbitrary. 
spectrum on the left was decomposed into contributions from the known $4 \tilde{\sigma}, 5 \tilde{\sigma}, 1 \tilde{\pi}$, and chemisorption-induced $\tilde{d}_{\pi}$ levels while the XA spectrum on the right shows the $2 \tilde{\pi}^{*}$ state, which is redshifted by $0.7 \mathrm{eV}$ compared to the gas phase $\mathrm{CO}$ [21]. Note that for the gas phase $\mathrm{CO}$ the $\tilde{d}_{\pi}$ is absent as it arises from hybridization with the metal substrate. The bottom panel (c) shows the corresponding spectrum averaged over the time delay range between 2 and $15 \mathrm{ps}$ after laser irradiation at an absorbed high optical laser fluence $\left(\sim 140 \mathrm{~J} / \mathrm{m}^{2}\right)$ where a laser pulse causes desorption of roughly a third of the adsorbed $\mathrm{CO}$ molecules [5]. Most notably we observe a decrease in the intensity of the $\tilde{d}_{\pi}$, accompanied by a shift towards higher emission energy of the occupied adsorbate states. Furthermore the increased intensity and blueshift of the $2 \tilde{\pi}^{*}$ indicate a weakening of the surface chemical bond due to transition into a weakly adsorbed precursor state [5]. The middle panel shows the spectra, again averaged over time delays between 2 and 15 ps, recorded with a slightly lower absorbed optical laser fluence $\left(\sim 100 \mathrm{~J} / \mathrm{m}^{2}\right)$, leading to desorption of $\sim 10 \%$ of a monolayer per laser shot [11]. In this case the laser pulse does not cause a high enough temperature rise for efficient transition into the precursor state [12]. These spectra differ significantly from spectra without an optical pump (negative delay), as well as from the high-fluence spectra at long time delays. The XA spectra that intrinsically sample all molecules show a decreased $2 \tilde{\pi}^{*}$ intensity together with a redshift, indicating stronger CO-metal $\pi$ interactions. By displaying two XE spectra using different probe energies, we highlight how this method can selectively study different subsets of the excited molecules. When we set the x-ray photon energy to absorb into the low-energy tail of the redshifted absorption resonance, we probe the changes induced by the low optical pump-laser fluence on the majority of the molecules: an increased $\tilde{d}_{\pi}$ intensity indicating stronger CO-metal $\pi$ interactions. Such spectral changes indicate that $\mathrm{CO}$ molecules move towards sites with higher coordination to the metal $[8,22]$ rather than populating the precursor state [5]. Setting the x-ray photon energy for absorption into the high-energy tail of the absorption spectrum instead, around the precursor state absorption resonance, we pick up a smaller subset of the molecules, which shows features towards the XE spectral signature of the precursor state, namely, a slightly decreased $\tilde{d}_{\pi}$ spectral weight and a small shift of the XE spectrum towards higher emission energies.

In order to quantify the ultrafast transient response of the $\mathrm{CO}$ to laser irradiation we analyze the time evolution of the $\mathrm{XA}$ and XE spectral features with a fitting procedure similar to that in Ref. [5] and present the results in Fig. 2. We observe spectral changes on two different time scales that can be connected with the dynamics of electrons and phonons in the metal substrate. Photo-induced reactions at metal surfaces are typically substrate mediated

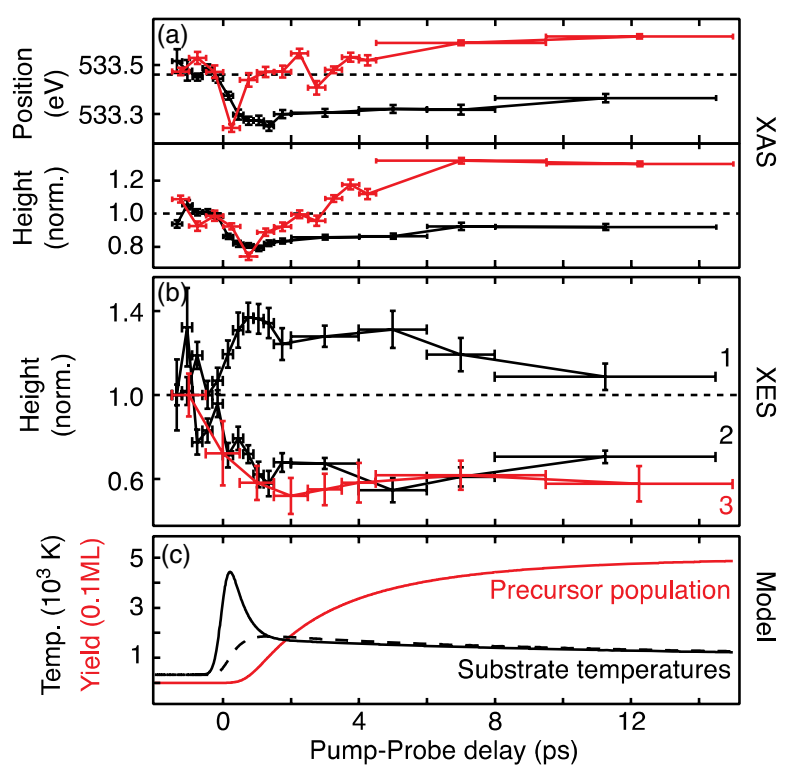

FIG. 2 (color online). Evolution of the absorption and emission spectra as a function of pump-probe delay for two different optical pump-laser fluences. Black/red lines are used in (a) and (b) for the lower $\left(100 \mathrm{~J} / \mathrm{m}^{2}\right) /$ higher $\left(140 \mathrm{~J} / \mathrm{m}^{2}\right)$ pump-laser fluence. (a) Position (top) and height (below) of the $2 \tilde{\pi}^{*}$ level extracted from a fitting procedure of XA spectra. (b) Height of the $\tilde{d}_{\pi}$ level extracted from the XES data. By choosing the X-ray photon energy, we can select different subsets of the $\mathrm{CO}$ molecules. Trace 1 stems from x-ray photons at the low-energy part of the $2 \tilde{\pi}^{*}$ resonance, trace 2 from $x$ rays probing the high-energy part and trace 3 from an intermediate x-ray energy but using a higher fluence optical laser excitation. (c) Calculated time evolution of the electron and phonon temperatures (black solid and dashed lines, respectively) together with the population of the precursor state (red line) obtained from an Arrhenius-like activation by the phonon temperature that transfers $\sim 50 \%$ of the molecules into the precursor state (red).

since the substrate absorbs the light much more efficiently than a single adsorbate layer [23-25]. Hot electrons are excited in the Ru substrate by the fs laser pulse, thermalize within $\sim 100$ fs [26], and may couple directly to the adsorbate vibrational degrees of freedom to initiate a reaction on a subpicosecond time scale. Alternatively the hot electrons heat the substrate phonon system that in turn excites the adsorbate dynamics [23]. Heating of the phonon system is slower than heating of the electron system and during the first picosecond after laser irradiation there is thus a strong nonequilibrium between the substrate electron and phonon temperatures; the time scale of the dynamics thus contains information about the reaction mechanism. The nonequilibrium between electron and phonon temperatures is demonstrated in Fig. 2(c), which shows a calculation of these temperatures within the two-temperature model $[16,17]$. In the present work we apply different optical laser fluences and use selective probing with different $\mathrm{x}$-ray photon energies to deconvolute processes driven by the two mechanisms. 
For the lower fluence (black), the XA $2 \tilde{\pi}^{*}$ position and height in, respectively, the top and bottom panels of Fig. 2(a) show the transient rapid change of the ensemble followed by a slower partial recovery, which matches the time scale of the changes in electron temperature plotted in Fig. 2(c). The temporal evolution of the spectroscopic features resembles the transient responses observed with vibrational spectroscopy for $\mathrm{CO}$ that were interpreted as a heating of the molecule leading to diffusion [1] or desorption [2-4] largely driven by frustrated rotations, that typically couple to the electron system on a subpicosecond time scale [1,27]. The apparent stronger $\pi$ interaction evidenced by the increasing $\tilde{d}_{\pi}$ intensity and the redshift with intensity lowering of the $2 \tilde{\pi}^{*}$ indicates that the majority of $\mathrm{CO}$ molecules moves away from the on-top site towards more highly coordinated bridge and hollow sites $[8,22]$. The observed quite large population change of hot chemisorbed $\mathrm{CO}$ molecules transferring towards higher coordination sites suggests a broad lateral distribution of vibrationally excited chemisorbed molecules consistent with a rather shallow corrugation in the lateral potential energy surface; this is similar to what has been determined by temperature-dependent line shape variations of, e.g., O $1 s$ spectra for $\mathrm{CO}$ on $\mathrm{Ni}(100)[28,29]$. This can also be interpreted as large amplitudes of coupled frustrated translations and rotations like those observed in the vibrational spectroscopy SFG experiments [1-4]. We conclude that the majority of molecules are vibrationally excited at this fluence but do not leave the chemisorbed state.

The high-fluence XAS data (red), on the other hand, initially show similar changes of the ensemble, but after a few picoseconds the transient changes are reversed and a completely different situation arises. Apparently a second, slower process that must be of different origin than the hotelectron excited vibrations is activated at the higher optical laser fluence. The time scale of this process suggests a phonon-mediated mechanism. This slower transient is related to the transition of the vibrationally excited chemisorbed $\mathrm{CO}$ molecules into a more weakly adsorbed precursor state where they reside for several picoseconds prior to desorption or readsorption [5].

How do the vibrationally hot chemisorbed state and the precursor state get populated? Are they mutually exclusive or are these populations formed simultaneously at the surface? These questions can be addressed using selective probing of subensembles of molecules in specific configurations, as demonstrated in Fig. 1(b).

Trace 1 in Fig. 2(b) shows the $\tilde{d}_{\pi}$ intensity in the lowfluence data when the x-ray photon energy is set to the lowenergy side of the XA resonance, where the intensity can be assumed to be mainly built up from the highly coordinated, vibrationally hot subset of CO molecules. Trace 2, on the other hand, was recorded with the same optical laser fluence as trace 1, but with the x-ray probe energy set to the high-energy side of the XA resonance, where the intensity is instead built up from weakly bonded $\mathrm{CO}$ molecules, thus selectively probing the contribution from the precursor state. This trace is similar to trace 3, which shows the ensemble average temporal evolution of the intensity of the $\tilde{d}_{\pi}$ states in the high-fluence XE spectra. In this case a decrease in the $\tilde{d}_{\pi}$ intensity is observed, which saturates below $70 \%$ of its original value after $\sim 2$ ps due to excitation of the molecules into the weakly adsorbed precursor state. This $\mathrm{x}$-ray probe energy dependence demonstrates the coexistence of the vibrationally hot and precursor adsorbed $\mathrm{CO}$ at the surface. Note that, although our selective spectroscopic method is able to pick up the signature of molecules in the precursor state at the lower optical laser fluence, their number is much smaller than those still chemisorbed at the surface at this fluence [12] [as shown by the dominating shift of the $2 \tilde{\pi}^{*}$ in Fig. 2(a)].

Previous work has suggested that both hot electrons and hot phonons couple strongly to $\mathrm{CO}$ desorption after laser excitation [11]. Here we can provide a microscopic interpretation of that finding by realizing that both the fast electron-driven vibrational excitation and slower excitation of surface phonons are necessary to laser induce desorption. A picture emerges where excitation of external adsorbate vibrations within the first picosecond dynamically weakens the surface chemical bond such that impulsive excitation from the substrate phonons after a few picoseconds may initiate the transition of a fraction of the molecules into the precursor state, which resides at a larger molecule-surface distance [5].

The picture of the two contributing processes is further substantiated by comparison with theoretical modeling. First we refer back to the time evolution of the substrate temperature modeled within the two-temperature model in Fig. 2(c). The electron temperature rises to a sharp maximum and decays within 1 ps to equilibrate with the phonon temperature, which at the equilibration time of $1.3 \mathrm{ps}$ reaches its maximum. Subsequently both temperatures decay slowly over several tens of picoseconds. The spectral changes attributed to vibrational excitation and diffusion reach a maximum within the first picosecond and fit well with the fast temperature rise of the electron system. The transition into the precursor state takes place on a longer time scale than the rise of the phonon temperature. The red lines in Fig. 2(c) show a modeled phonon-driven Arrhenius transition leading to a total yield of $0.5 \mathrm{ML}$ in the precursor state as an example. This modeled transition takes place on the same time scale as the experimentally measured transition into the precursor state and supports the notion of a phonon-mediated transition into the precursor state. Although a similar transient might also be consistent with a weakly electron-mediated process [30], our AIMD simulations [13] support the involvement of substrate phonons in the transition into the precursor state. The simulations correspond to the experimental situation at delays longer than the electron-phonon equilibration time of 


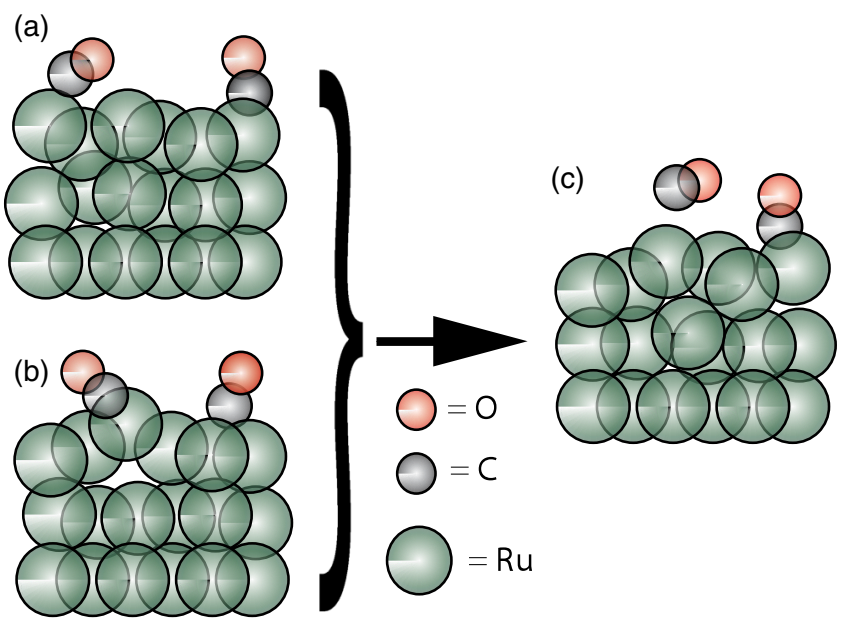

FIG. 3 (color online). Snapshots taken from a $3000 \mathrm{~K}$ AIMD simulation containing two $\mathrm{CO}$ molecules on a $\mathrm{Ru}(0001)$ slab (a), (b) to illustrate examples of processes taking place after equilibration between the electron and phonon systems $(\sim 1 \mathrm{ps}$ after the optical laser pump) that eventually leads to $\mathrm{CO}$ desorption (c). $\mathrm{CO}$ desorption is mediated by an interplay between a highly mobile substrate, diffusion of adsorbates through translational motion, which is activated by frustrated rotations, and furthermore also enhanced by collisions with the distorted substrate.

$\sim 1$ ps when both the electron- and phonon-induced processes contribute to the overall dynamics. Figure 3 shows selected AIMD snapshots representing the relevant processes leading to CO desorption; because of the high cost of these simulations, too few trajectories for reliable statistics could be run. Similar to the case of CO on Pt(533) [1] our simulations show that translational motion is activated by the frustrated rotational vibrations, visualized by the severely tilted $\mathrm{CO}$ molecules in snapshot (a) in Fig. 3. Note the highly distorted $\mathrm{Ru}$ atom positions [see, e.g., snapshot (b)] and the movement in the substrate that results in numerous collisions with the adsorbate throughout the simulations. After several picoseconds in the simulations we observe $\mathrm{CO}$ molecules leaving the chemisorbed state at the metal surface [snapshot (c)]. This process is seemingly mediated by frustrated rotations and collisions with the highly mobile and disordered substrate (i.e., hot phonons).

In conclusion we have demonstrated ultrafast resonant $\mathrm{x}$-ray emission and absorption as tools for selectively studying the valence electronic structure of different coadsorbed transient species during surface reactions. We have applied these techniques to the case of $\mathrm{CO}$ desorption, where we show the coexistence of both vibrationally excited chemisorbed $\mathrm{CO}$ and of $\mathrm{CO}$ in a weakly adsorbed precursor state, and selectively probed the electronic structure of these different transient species. We established a two-step mechanism leading to $\mathrm{CO}$ desorption after an optical laser excitation. Subpicosecond electron-driven dynamics lead to excitation of $\mathrm{CO}$ external vibrational modes and diffusion, which in combination with slower, phonondriven ( $>2 \mathrm{ps}$ ) dynamics leads to population of the precursor state prior to desorption. This possibility of time-resolved selective probing of the electronic structure provides a unique opportunity for further detailed studies of transient phenomena during reactions in heterogeneous catalysis.

This research was carried out on the SXR Instrument at the Linac Coherent Light Source (LCLS) at the SLAC National Accelerator Laboratory. The SXR Instrument is funded by a consortium whose membership include the LCLS, Stanford University through the Stanford Institute for Materials Energy Sciences (SIMES), Lawrence Berkeley National Laboratory (LBNL), University of Hamburg through the BMBF priority program FSP 301, and the Center for Free Electron Laser Science (CFEL). The LCLS is funded by the U.S. Department of Energy's Office of Basic Energy Sciences. We gratefully acknowledge the support of the LCLS staff. This work was further supported by the Swedish research council (VR), the Alexander von Humboldt foundation and the VolkswagenStiftung. The simulations were performed using resources provided by the Swedish National Infrastructure for Computing (SNIC) at the Center for Scientific and Technical Computing LUNARC, Lund University.

*henrik.ostrom@fysik.su.se

[1] E. H. G. Backus, A. Eichler, A. W. Kleyn, and M. Bonn, Science 310, 1790 (2005).

[2] M. Bonn, C. Hess, S. Funk, J. H. Miners, B. N. J. Persson, M. Wolf, and G. Ertl, Phys. Rev. Lett. 84, 4653 (2000).

[3] F. Fournier, W. Zheng, S. Carrez, H. Dubost, and B. Bourguignon, J. Chem. Phys. 121, 4839 (2004).

[4] K. Inoue, K. Watanabe, and Y. Matsumoto, J. Chem. Phys. 137, 024704 (2012).

[5] M. Dell'Angela et al., Science 339, 1302 (2013).

[6] A. Nilsson and L. G. M. Pettersson, Surf. Sci. Rep. 55, 49 (2004).

[7] A. Nilsson and L. G. M. Pettersson, in Chemical Bonding at Surfaces and Interfaces, edited by A. Nilsson, L. G. M. Pettersson, and J.K. Nørskov (Elsevier, Amsterdam, 2008), pp. 57.

[8] A. Föhlisch, M. Nyberg, J. Hasselström, O. Karis, L. G. M. Pettersson, and A. Nilsson, Phys. Rev. Lett. 85, 3309 (2000).

[9] A. Nilsson, M. Weinelt, T. Wiell, P. Bennich, O. Karis, N. Wassdahl, J. Stöhr, and M. G. Samant, Phys. Rev. Lett. 78, 2847 (1997).

[10] W.F. Schlotter et al., Rev. Sci. Instrum. 83, 043107 (2012).

[11] J. Gladh, T. Hansson, and H. Öström (unpublished).

[12] See Supplemental Material at http://link.aps.org/ supplemental/10.1103/PhysRevLett.110.186101 for theoretical details.

[13] Calculations were performed using the GPAW code with a grid spacing of $0.2 \AA$, a $(4 \times 4 \times 1) k$-point sampling, a $\left(5.5 \times 4.8 \times 17.4 \AA^{3}\right)$ unit cell, and a time step of $2 \mathrm{fs}$. $)$. 
[14] B. Hammer, L. B. Hansen, and J. K. Nørskov, Phys. Rev. B 59, 7413 (1999).

[15] M. Dion, H. Rydberg, E. Schröder, D. C. Langreth, and B. I. Lundqvist, Phys. Rev. Lett. 92, 246401 (2004).

[16] S. I. Anisimov, B. L. Kapeliovich, and T. L. Perel'man, Sov. Phys. JETP 39, 375 (1974).

[17] M. I. Kaganov, I. M. Lifshitz, and L. V. Tanatarov, Sov. Phys. JETP 4, 173 (1957).

[18] A. Föhlisch, P. Bennich, J. Hasselström, O. Karis, A. Nilsson, M. Nyberg, L. Triguero, and L.G.M. Pettersson, J. Chem. Phys. 112, 1946 (2000).

[19] A. Föhlisch, J. Hasselström, P. Bennich, N. Wassdahl, O. Karis, A. Nilsson, L. Triguero, M. Nyberg, and L. G. M. Pettersson, Phys. Rev. B 61, 16229 (2000).

[20] A. Föhlisch, W. Wurth, M. Stichler, C. Keller, and A. Nilsson, J. Chem. Phys. 121, 4848 (2004).

[21] P. Skytt, P. Glans, K. Gunnelin, J. Guo, J. Nordgren, Y. Luo, and H. Ågren, Phys. Rev. A 55, 134 (1997).
[22] H. Tillborg, A. Nilsson, N. Mårtensson, and J. N. Andersen, Phys. Rev. B 47, 1699 (1993).

[23] M. Bonn, S. Funk, C. Hess, D. N. Denzler, C. Stampfl, M. Scheffler, M. Wolf, and G. Ertl, Science 285, 1042 (1999).

[24] J. A. Misewich, T. F. Heinz, and D. M. Newns, Phys. Rev. Lett. 68, 3737 (1992).

[25] F. M.Zimmermann and W. Ho, Surf. Sci. Rep. 22, 127 (1995).

[26] M. Lisowski, P. A. Loukakos, U. Bovensiepen, J. Stähler, C. Gahl, and M. Wolf, Appl. Phys. A 78, 165 (2004).

[27] M. Head-Gordon and J. C. Tully, Phys. Rev. B 46, 1853 (1992).

[28] H. Antonsson, A. Nilsson, N. Mårtensson, I. Panas, and P. Siegbahn, J. Electron Spectrosc. Relat. Phenom. 54-55, 601 (1990).

[29] A. Nilsson and N. Mårtensson, Solid State Commun. 70, 923 (1989).

[30] S. Funk, M. Bonn, D. N. Denzler, C. Hess, M. Wolf, and G. Ertl, J. Chem. Phys. 112, 9888 (2000). 